NASA Technical Memorandum 106194

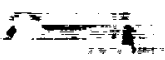

$$
1 N-35
$$

\title{
Fuzzy Logic Particle Tracking Velocimetry
}

Mark P. Wernet

Lewis Research Center Cleveland, Ohio
(NASA-TM-106194) FUZZY LOGIC

PARTICLE TRACKING VELOCIMETRY

(NASA) $10 \mathrm{P}$
N93-27027

Unclas

$63 / 35 \quad 0167914$

Prepared for the

Optical Diagnostics in Fluid and Thermal Flow

sponsored by the Society of Photo-Optical Instrumentation Engineers

San Diego, California, July 11-16, 1993 


\title{
Fuzzy logic particle tracking velocimetry
}

\author{
Mark P. Wernet \\ National Aeronautics and Space Administration \\ Lewis Research Center \\ Cleveland, $\mathrm{OH} 44135$
}

\begin{abstract}
Fuzzy logic has proven to be a simple and robust method for process control. Instead of requiring a complex model of the system, a user defined rule base is used to control the process. In this paper the principles of fuzzy logic control are applied to Particle Tracking Velocimetry (PTV). Two frames of digitally recorded, single exposure particle imagery are used as input. The fuzzy processor uses the local particle displacement information to determine the correct particle tracks. Fuzzy PTV is an improvement over traditional PTV techniques which typically require a sequence $(>2)$ of image frames for accurately tracking particles. The fuzzy processor executes in software on a PC without the use of specialized array or fuzzy logic processors. A pair of sample input images with roughly 300 particle images each results in more than 200 velocity vectors in under 8 seconds of processing time.
\end{abstract}

\section{INTRODUCTION}

The control of complex processes has been aided by the development of fuzzy control systems. Fuzzy control systems have been used to control traffic flow, appliances, and even subways for optimal energy efficiency and passenger comfort. ${ }^{1}$ Fuzzy logic employs a tolerance for imprecision to achieve system control. ${ }^{2}$ An exact model for the system inputs and outputs is not required. Fuzzy inference control utilizes membership functions and a rule base developed by the user to process information. The physical mechanisms underlying the process are impertinent to the controller. The process of identifying and tracking particles in a flow is a good candidate for fuzzy control since the procedure is not clear cut, but involves some gray area decisions.

Particle Tracking Velocimetry (PTV) is a planar velocity measurement technique whereby the positions of particles entrained in a fluid are recorded over a planar region at more than one instance in time. ${ }^{3}$ A light sheet is used to illuminate the particles in the fluid. The exposures can be recorded either on a single frame, or on independent frames. When independent frames are used, the velocity vector direction information is easily determined. However, mechanical shifting between exposures recorded on a single frame also reveals direction information. ${ }^{4}$

Double exposure, single frame imagery are more commonly processed via correlation techniques and require high data densities. ${ }^{4}$ The correlation techniques rely on the local average particle displacement to determine the net velocity over the interrogated region. PTV techniques typically require more than two exposures and can perform well in both high and low data density cases. 5 .6 The PTV techniques require more time history information in order to make an accurate identification of a valid particle track, which requires more data storage and/or higher framing rate cameras.

This paper describes the application of fuzzy inference techniques to track individual particles recorded on a pair of single exposure frames. PTV is a good candidate for fuzzy control since the particle trajectories are never identical and the velocity field varies spatially across the image plane. Local particle displacement information is used to identify candidate particle tracks. The fuzzy inference processor is used to determine the most probable particle trajectories based on common sense rules that an observer would use to identify particle tracks. This is the first known application of fuzzy control to PTV. Similar work has been done in applying neural networks to particle tracking. ${ }^{7}$

A sample flow field is recorded and reduced using the Fuzzy PTV reduction technique. The fuzzy logic PTV runs in software on a PC, without specialized array processors. A sample data set was reduced in under 8 seconds yielding over 200 velocity vectors. 


\section{PARTICLE TRACKING VELOCIMETRY}

The PTV technique described herein employs direct digital recording of the particle images onto a CCD array. Two successive single exposure video fields are acquired from the CCD camera. The two image fields are independently processed to determine the particle image centroids. The particle image amplitude and shape information are used to compute the intensity weighted mean particle location. The centroid locations are stored and used to determine candidate displacement vectors. Velocity vectors are calculated by dividing the displacement vector by the inter-exposure time.

The particle centroids on the initial Frame \#1 are used as starting points for possible particle displacements. The user specifies a search region radius, $R_{s}$ typically 10-20 pixels, to search for Frame \#2 particles. Each Frame \#2 particle within a radius $R_{s}$ from the initial particle centroid is a candidate displacement vector. All possible displacements of the initial particle to the second particle locations within the search region are recorded and stored as lists of candidate displacement vectors for that initial particle. Hence, for high data density areas, many initial particles may be competing for the same second exposure particle centroids. At this stage in the processing, the vector field is very convoluted and noisy. The fuzzy inference processor operates on these lists of candidate displacement vectors to determine the most likely displacement vector for each initial particle centroid location.

The list of vectors for each initial particle is compared to all other initial particle displacement vector lists to determine if there is any commonality. If two separate initial particles do claim the same second particle, then all possible vector pairs between these two initial particles are compared. The main assumption is that if two initial particles are close enough to interact (claim the same second particle) then the pair of vectors that look the most similar (in direction and magnitude) must be the correct pair of displacement vectors for the two separate initial particles. This assumption also holds for tertiary and higher interactions.

\section{FUZZY LOGIC PROCESSOR APPLIED TO PTV}

There are four inputs to the Fuzzy PTV processor for each vector pair: distance between the vector midpoints in pixels (Sep); average vector magnitude (Mag); difference in vector magnitudes (MagDif); and the sum of the squares of the differences of the $x$-and $y$-components of the two velocity vectors (Delta). The first and third measures, when combined, act as a velocity gradient measure. The last measure operates in the opposite manner to the dot product. This measure is small when the vectors are similar in magnitude and direction and large when they are different. Each input measure is assigned to a fuzzy set, where the degree of membership for each element in the set varies between 0 and 1 . Standard 25-50\% overlapping triangular input membership functions are used as shown in figure 1 . The degrees of membership for each input are processed through a rule base of "IF...THEN" blocks. The rule base defines an output fuzzy set. For a given vector pair, up to 16 rules may fire depending on the number of unique combinations of membership values. In lieu of the more common centroiding technique, the Fuzzy PTV processor output is computed via the singleton technique with a weighted average, which is computationally simpler. ${ }^{8}$

The Fuzzy PTV rule base is shown in matrix format in table 1. Each input measure has three elements in its fuzzy set: (Small, Med, Large). Every fuzzy set has a corresponding set of membership functions. The membership functions assign a numeric weight, or degree of membership $\mu$, for all elements in each input measure's respective fuzzy set. The number of non-zero memberships depends on the amount of overlap of the membership functions. The midpoints of the membership functions are: Sep_Mid=R $;$ Mag_Mid=Rs/2;MagDif_Mid=5 pixels; Delta_Mid=5 pixels. $R_{s}$ is the search region size in pixels, which is nominally 20 pixels.

The input measures are computed for each vector pair analyzed. The fuzzy processor output from the rule base is the fuzzy set of confidence in the vector pair. The set of confidence singletons and their associated numerical weights are: $\{$ High=0.8; Med=0.5; Low =0.1; None=0\}. For example, in table 1 with $\mathrm{Sep}=S$ mall, Mag=Med, MagDif=Small, and Delta=Med yields Conf_Out=Med. 


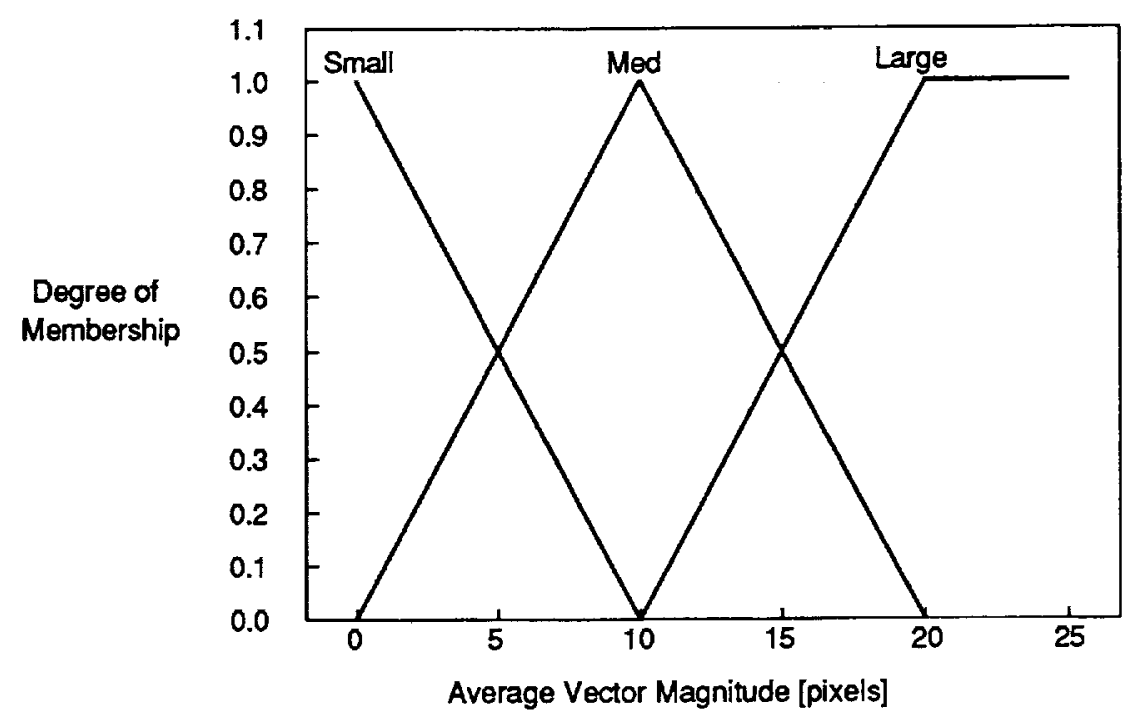

Figure 1: Input membership functions with $50 \%$ overlap for the average vector magnitude fuzzy set.

\begin{tabular}{|c|c|c|c|c|c|c|c|c|c|}
\hline \multirow{2}{*}{ Sep Small } & \multicolumn{3}{|c|}{ MagDif Small } & \multicolumn{3}{c|}{ MagDif Med } & \multicolumn{3}{c|}{ MagDif Large } \\
\cline { 2 - 9 } & $\begin{array}{c}\text { Mag } \\
\text { small }\end{array}$ & $\begin{array}{c}\text { Mag } \\
\text { Med }\end{array}$ & $\begin{array}{c}\text { Mag } \\
\text { Large }\end{array}$ & $\begin{array}{c}\text { Mag } \\
\text { small }\end{array}$ & $\begin{array}{c}\text { Mag } \\
\text { Med }\end{array}$ & $\begin{array}{c}\text { Mag } \\
\text { Large }\end{array}$ & $\begin{array}{c}\text { Mag } \\
\text { small }\end{array}$ & $\begin{array}{c}\text { Mag } \\
\text { Med }\end{array}$ & $\begin{array}{c}\text { Mag } \\
\text { Large }\end{array}$ \\
\hline Delta Small & High & High & High & High & Med & Med & Med & Med & Low \\
\hline Delta Med & High & Med & Low & Med & Med & Med & Low & Low & Low \\
\hline Delta Large & Med & Low & Low & Low & Low & Low & Low & Low & Low \\
\hline
\end{tabular}

\begin{tabular}{|c|c|c|c|c|c|c|c|c|c|}
\hline \multirow{2}{*}{ Sep Med } & \multicolumn{3}{|c|}{ MagDif Small } & \multicolumn{3}{c|}{ MagDif Med } & \multicolumn{3}{c|}{ MagDif Large } \\
\cline { 2 - 9 } & $\begin{array}{c}\text { Mag } \\
\text { small }\end{array}$ & $\begin{array}{c}\text { Mag } \\
\text { Med }\end{array}$ & $\begin{array}{c}\text { Mag } \\
\text { Large }\end{array}$ & $\begin{array}{c}\text { Mag } \\
\text { small }\end{array}$ & $\begin{array}{c}\text { Mag } \\
\text { Med }\end{array}$ & $\begin{array}{c}\text { Mag } \\
\text { Large }\end{array}$ & $\begin{array}{c}\text { Mag } \\
\text { small }\end{array}$ & $\begin{array}{c}\text { Mag } \\
\text { Med }\end{array}$ & $\begin{array}{c}\text { Mag } \\
\text { Large }\end{array}$ \\
\hline Delta Small & High & Med & Med & Med & Med & Low & Med & Low & Low \\
\hline Delta Med & Med & Med & Low & Med & Med & Low & Low & Low & Low \\
\hline Delta Large & Low & Low & Low & Low & Low & Low & Low & Low & Low \\
\hline
\end{tabular}

\begin{tabular}{|c|c|c|c|c|c|c|c|c|c|}
\hline \multirow{2}{*}{ Sep Large } & \multicolumn{3}{|c|}{ MagDif Small } & \multicolumn{3}{c|}{ MagDif Med } & \multicolumn{3}{c|}{ MagDif Large } \\
\cline { 2 - 10 } & $\begin{array}{c}\text { Mag } \\
\text { small }\end{array}$ & $\begin{array}{c}\text { Mag } \\
\text { Med }\end{array}$ & $\begin{array}{c}\text { Mag } \\
\text { Large }\end{array}$ & $\begin{array}{c}\text { Mag } \\
\text { small }\end{array}$ & $\begin{array}{c}\text { Mag } \\
\text { Med }\end{array}$ & $\begin{array}{c}\text { Mag } \\
\text { Large }\end{array}$ & $\begin{array}{c}\text { Mag } \\
\text { small }\end{array}$ & $\begin{array}{c}\text { Mag } \\
\text { Med }\end{array}$ & $\begin{array}{c}\text { Mag } \\
\text { Large }\end{array}$ \\
\hline Delta Small & Med & Med & Low & Med & Low & Low & Low & Low & Low \\
\hline Delta Med & Low & Low & Low & Low & Low & Low & Low & Low & Low \\
\hline Delta Large & Low & Low & Low & Low & Low & Low & Low & Low & None \\
\hline
\end{tabular}

Table 1: Rule base for Fuzzy PTV processor. Each unique combination of inputs defines an output element of Conf_Out.

All unique combinations of the degrees of membership for each fuzzy set for each input measure are combined via a fuzzy "AND", which is the minimum value of the four membership values. The actual confidence level in the vector pair is computed by taking the product of the fired rule output value (Conf_Out) and the fuzzy "AND" of the degree of memberships. A sum of these products is computed over all of the fired rules. The total is normalized by the sum of the minimum degree of 
membership values for each rule fired. The output of the fuzzy processor represents the crisp estimate of the confidence in the velocity vector pair, ranging from 0 (low confidence) to 1 (high confidence).

For example, given two pairs of vectors formed from two initial points competing for the same second particle as shown in figure 2 and in table 2:

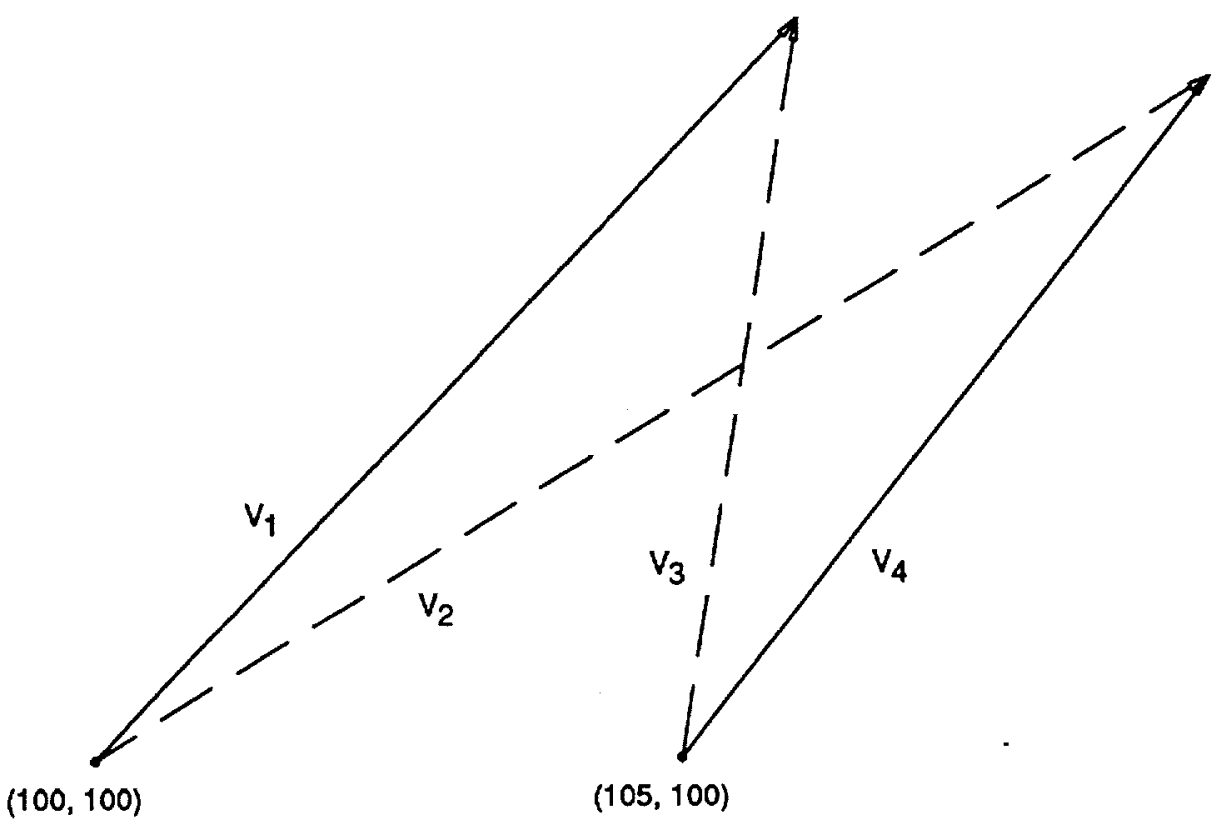

Figure 2: Two pairs of interacting vectors with separate initial points. Dashed line indicates incorrect vectors, solid line denotes correct vectors.

\begin{tabular}{|c|c|c|c|c|}
\cline { 2 - 5 } \multicolumn{1}{c|}{} & $\mathrm{X}_{\mathrm{i}}$ & $\mathrm{Y}_{\mathrm{i}}$ & $\overline{\mathrm{V}}$ [pixels] & $\theta^{\circ}$ \\
\hline $\mathrm{V}_{1}$ & 100 & 100 & 8.5 & 45 \\
\hline $\mathrm{V}_{2}$ & 100 & 100 & 11 & 30 \\
\hline $\mathrm{V}_{3}$ & 105 & 100 & 6 & 81 \\
\hline $\mathrm{V}_{4}$ & 105 & 100 & 7.1 & 51 \\
\hline
\end{tabular}

Table 2: Sample data for an interacting pair of vectors.

We find the following combinations, and their respective input measures to the membership functions:

\begin{tabular}{|c|c|c|c|c|}
\cline { 2 - 5 } \multicolumn{1}{c|}{} & Sep & Mag & MagDif & Delta \\
\hline $\mathrm{V}_{1} \mathrm{~V}_{3}$ & 0 & 7.3 & 2.5 & 25 \\
\hline $\mathrm{V}_{1} \mathrm{~V}_{4}$ & 4.3 & 7.8 & 1.4 & 2.5 \\
\hline $\mathrm{V}_{2} \mathrm{~V}_{3}$ & 1.7 & 8.5 & 5 & 71.6 \\
\hline $\mathrm{V}_{2} \mathrm{~V}_{4}$ & 2.6 & 9.0 & 3.9 & 24.4 \\
\hline
\end{tabular}

\begin{tabular}{|c|}
\hline Confidence \\
\hline 0.24 \\
\hline 0.67 \\
\hline 0.11 \\
\hline 0.24 \\
\hline
\end{tabular}

Table 3: Fuzzy processor input measures and outputs for sample vector pair combinations 
Looking at the first case, $V_{1} V_{3}$ has the fuzzy set memberships: $\operatorname{Sep}\left\{\mu_{\mathrm{Small}}=0.875, \mu_{\mathrm{Med}}=0, \mu_{\mathrm{Lage}}=0\right\}$; $\operatorname{Mag}\left\{\mu_{\text {Small }}=0.276, \mu_{\text {Med }}=0.724, \mu_{\text {Large }}=0\right\} ; \operatorname{MagDif}\left(\mu_{\text {Small }}=0.504, \mu_{\text {Med }}=0, \mu_{\mathrm{Larg}}=0\right\} ;$ Delta $\left(\mu_{\text {Small }}=0, \mu_{\text {Med }}=0, \mu_{\text {Lage }}=1\right\}$. With these fuzzy sets the following rules are fired:
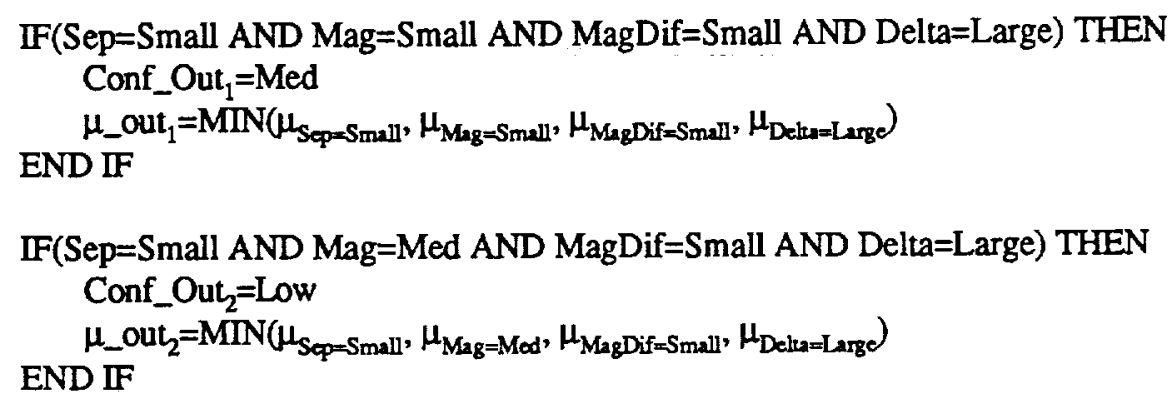

The fuzzy "AND" of the memberships in rule $\# 1$ gives $\mu_{-}$out ${ }_{1}=0.276$ and rule $\# 2$ gives $\mu_{-}$out $t_{2}=0.504$. The fuzzy processor output for this vector pair is given by:

$$
\begin{gathered}
\text { Confidence }=\frac{\sum_{i=1}^{N_{R}} \mathrm{Conf}_{-} \mathrm{Out}_{i} \cdot \mu_{-} \text {out }_{i}}{\sum_{i=1}^{N_{R}} \mu_{-} \mathrm{out}_{i}} \\
\text { Confidence }=\frac{0.5 \cdot 0.276+0.1 \cdot 0.504}{0.276+0.504}=0.24
\end{gathered}
$$

where $\mathrm{N}_{R}$ is the number of fired rules. The fuzzy processor output confidence level in each vector pair is shown in table 3 . For the vector pairs listed, the $\mathrm{V}_{1} \mathrm{~V}_{4}$ pair has the highest confidence level of all the combinations. Initially, all of the vectors are assigned a confidence level of 0 . The computed confidence level for the vector pair is compared against the current confidence level for each vector in the pair, and the maximum confidence value is stored for each vector. When all of the interacting vector pairs have been analyzed, the list of candidate vectors for each initial point will have confidence levels relative to one another. The vector in the list with the highest confidence level is assumed to be the most probable vector for the current initial point, and moved to the top of the list.

A second pass through the data is performed to analyze non-interacting velocity vectors lists. An non-interacting vector is defined as a vector with multiple candidate vectors for a single initial point, but does not share a common second particle centroid with any other vector. The overall flow field pattern is used to determine the correctness of these outlying velocity vectors. The fuzzy processor is used to compare each vector in the list to all of the other previously analyzed velocity vectors in the flow field. Again, the vector with the highest confidence value in the list is assumed to be the correct vector.

A final pass through the data is performed in which the highest confidence velocity vectors for each initial particle centroid are compared. All of the confidence levels are reset to 0 . Each remaining valid vector is compared to all other valid vectors in the flow field. The resulting confidence level for each vector is then compared to a fixed threshold level. Only vectors with confidence levels above the set threshold level are considered valid.

\section{EXPERIMENT}

The experimental setup is shown in figure 3 and discussed in detail in reference 9. The test chamber consists of a $10 \mathrm{~cm}$ diameter by $5 \mathrm{~cm}$ deep cylinder bored into a $12.7 \mathrm{~cm}$ square by $6.4 \mathrm{~cm}$ high plexiglass block. The reservoir was filled with 10 centistoke silicone oil and seeded with $12 \mu \mathrm{m}$ pliolite particles. The flow was driven by the tubular heater in the center of the reservoir. The data were recorded onto a VCR using a gated intensified CCD camera. A large memory buffer 
framegrabber was used to digitize the signals from the VCR at $640 \times 480$ pixels by 8 bits. Two successive video fields were acquired from a single camera for this low velocity flow.

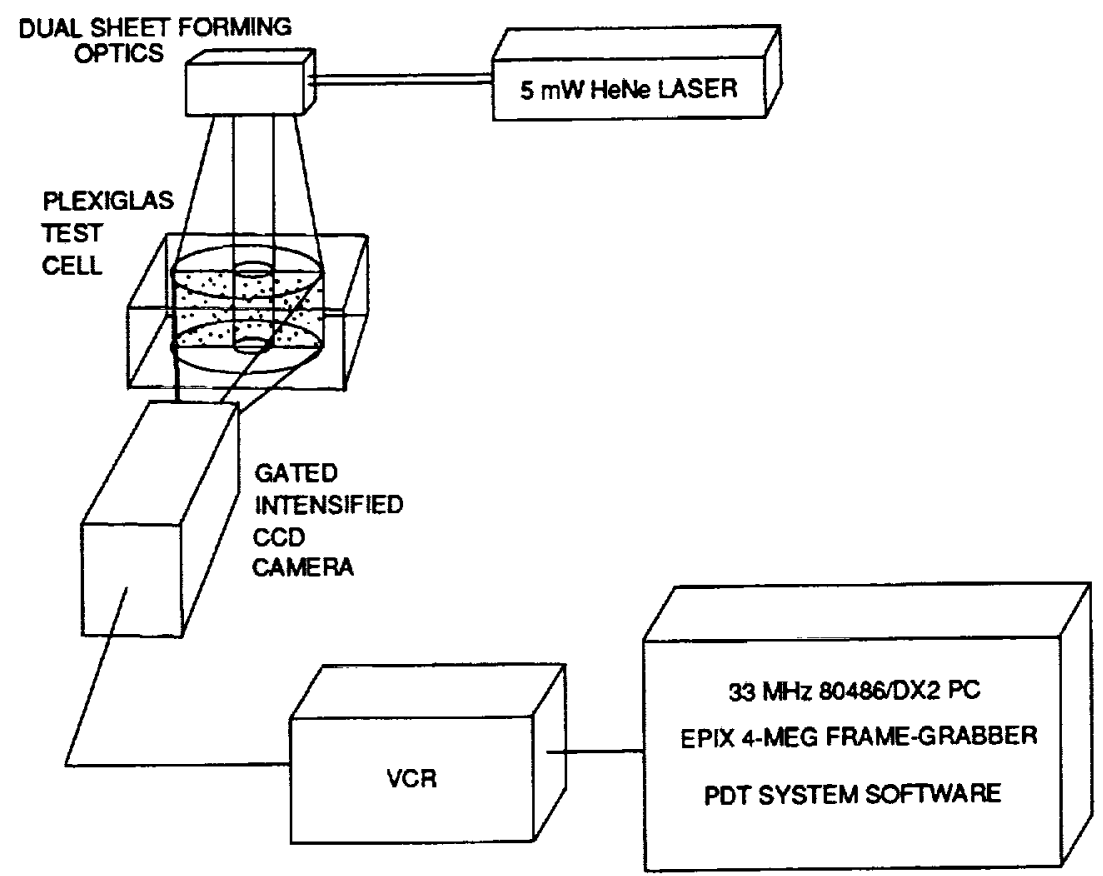

Figure 3: Schematic layout of experimental setup

\section{RESULTS AND DISCUSSION}

The flow field was allowed to reach steady state before the particle image data were acquired. The time interval between the two video fields was 1 second. The images were processed with a background threshold level of 10 grey levels. The particle centroids were determined via an intensity weighted mean calculation of the identified particle images in the field. The centroid processed images contained 316 and 318 particle images, respectively. The centroid error for this experimental configuration has been previously determined to be \pm 0.25 pixels. ${ }^{10}$

The information input into the fuzzy processor is a list of candidate velocity vectors for each initial particle centroid. The number of interacting velocity vectors is affected by the data density and the search region size $R_{s}$. The 1141 identified candidate velocity vectors are shown in figure 4.

The output from the fuzzy processor is shown in figure 5. The resulting vector field map contains 209 velocity vectors. The total processing time was 7.6 seconds. The success rate of vector identification to number of particle image centroids in the image is $66 \%$. The minimum measured velocity vector displacement was 0.25 pixels and the maximum measured displacement was 17.7 pixels. The corresponding minimum and maximum velocities were 0.04 and $2.87 \mathrm{~mm} / \mathrm{s}$ respectively. The measured velocity dynamic range was 70:1. There are 4 incorrectly identified velocity vectors shown in figure 5 . The region near the fluid surface close to the heater is a region of high acceleration. The particle displacements in this region are on the order of or greater than 20 pixels. Many of the unsuccessful matchings have occurred in this region. The higher velocity region of the flow could be examined by decreasing the interframe acquisition time.

Fuzzy PTV is similar to cross-correlation techniques used in normal PIV data reduction. Seeding densities comparable to those used for correlation data reduction yield good results. The local displacement information is used to determine the correct vector displacement. However, individual velocity vectors are detected, not the average displacement over an interrogation region. Hence, Fuzzy PTV can have better spatial resolution than correlation techniques. PTV 
techniques can have similar or higher accuracies than correlation techniques when averaged over similar sized sub-region areas. ${ }^{10}$

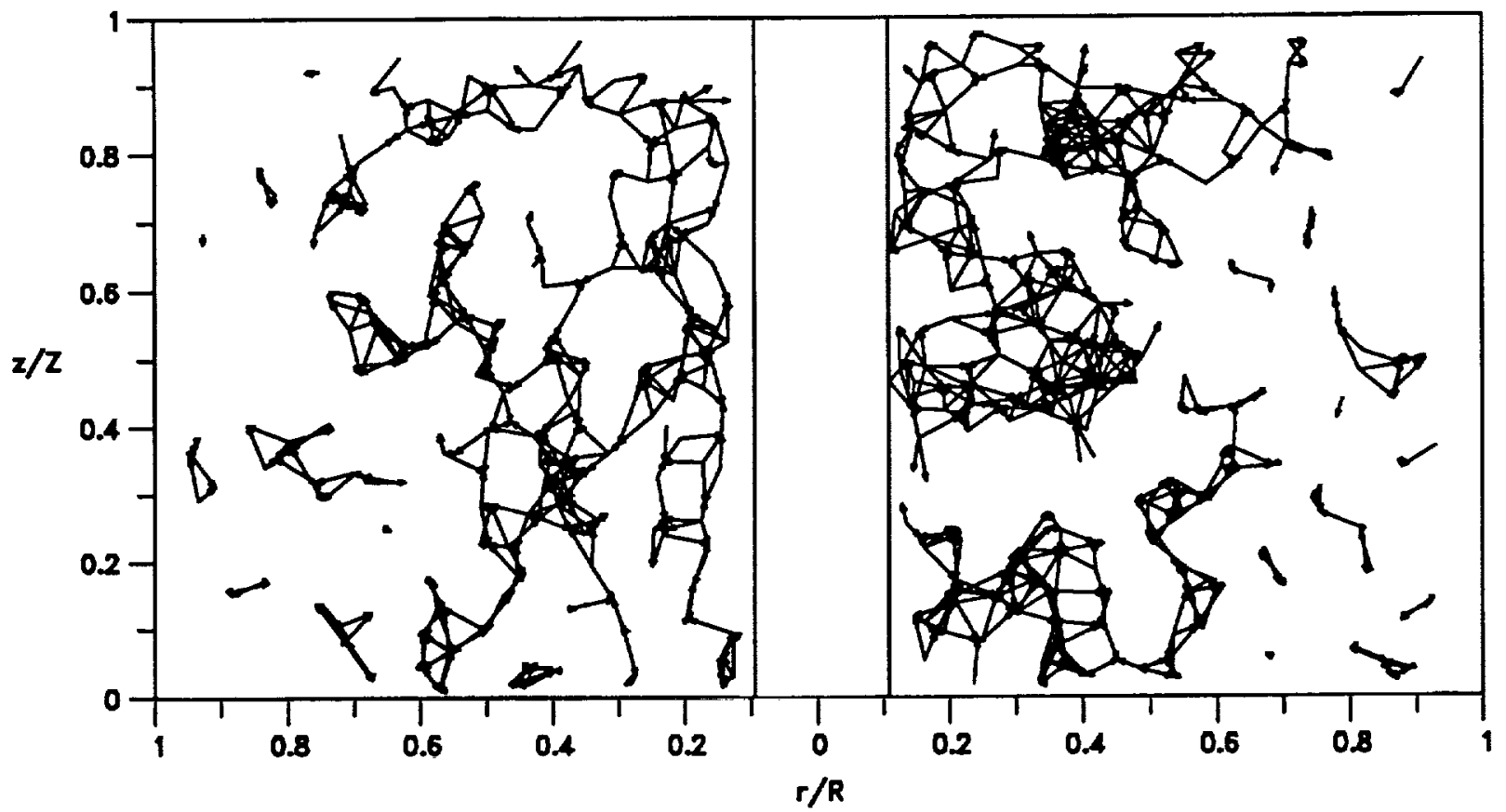

Figure 4: Plot of all candidate velocity vectors for each initial centroid location.

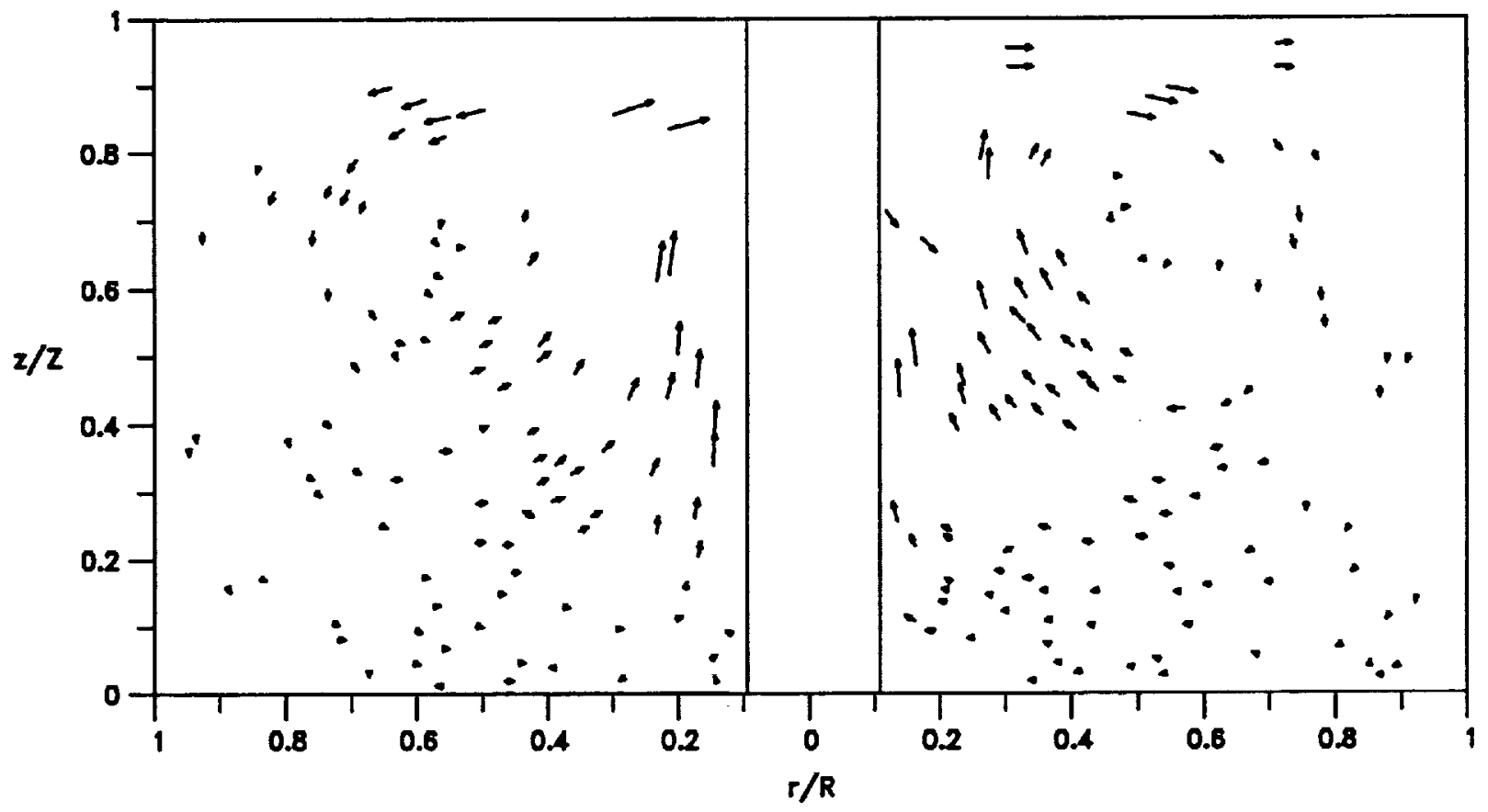

Figure 5: Plot of velocity vector field output from fuzzy processor. 
Future improvements to the fuzzy processor will be the inclusion of particle image shape and amplitude information as additional input measures. Inclusion of the particle image attributes further improves the robustness of the fuzzy tracking processor. The individual particle image shape and amplitude information can also be used to estimate the detected velocity vector accuracy as described in reference 10.

\section{CONCLUSIONS}

The application of fuzzy logic principles to the task of tracking particles recorded on two separate image frames has been demonstrated. The rule base developed is very simple and the fuzzy processor executed in software on a PC with good performance. Fuzzy techniques are simple to apply since no model of the flow field is required. Once the processor is established, no training is required as in neural network techniques.

Fuzzy PTV is an attractive altemative to correlation techniques since no specialized processing hardware is required for data reduction or analysis. Direct digital recording simplifies the data processing. Fuzzy PTV requires only two independent image frames compared to traditional PTV techniques which require a sequence of images. Further enhancements to the fuzzy processor will increase its robustness and provide for individual velocity vector error estimates.

\section{REFERENCES}

1. D. I. Brubaker and C. Sheerer, "Fuzzy-logic system solves control problem", Electronic Design News, pp. 121-127, June 18, 1992.

2. L. A. Zadeh, "Fuzzy sets", Information and Control, Vol. 8, pp. 338-352, 1965.

3. R. J. Adrian, "Multi-point optical measurements of simultaneous vectors in unsteady flow - a review", Int. J. of Heat and Fluid Flow, Vol 7, pp. 127-145, 1986.

4. R. J. Adrian, "Image shifting technique to resolve directional ambiguity in double-pulsed velocimetry", Appl. Opt., Vol 25, pp. 3855-3858, 1986.

5. M. P. Wernet and A. D. Pline, "Particle image velocimetry for the surface tension driven convection experiment using a particle displacement tracking technique", Proceeding $4^{\text {th }}$ Int. Conference on Laser Anemometry Advances and Applications, Cleveland, Ohio, pp. 326-335, 1991.

6. I. D. Haynes and J. T. Turner, "High speed on-line whole field processing of complex flows", Proceedings of the $6^{\text {th }}$ Int. Symposium on Applications of Laser Techniques to Fluid Mechanics, Lisbon, Portugal, pp. 3.3.1-3.3.6, 1992.

7. A. Cenedese, G.P. Romano, A. Paglialunga, M. Terlizzi, "Neural net for trajectories recognition in a flow", Proceedings of the $6^{\text {th }}$ Int. Symposium on Applications of Laser Techniques to Fluid Mechanics, Lisbon, Portugal, pp. 27.1.1-27.1.5, 1992.

8. D. I. Brubaker, "Fuzzy--Iogic basics: intuitive rules replace complex math", Electronic Design News, pp. 111-116, June 18, 1992.

9. A. D. Pline, T. P. Jacobson, J. S. Wanhainen, D. A. Petrarca, "Hardware development for the surface tension driven convection experiment", J. Spacecraft and Rockets, Vol. 27, pp. 312-317, 1990.

10. M. P. Wernet and A. Pline, "Particle displacement tracking technique and Cramer-Rao lower bound error in centroid estimates from CCD imagery", Proceedings of $13^{\text {th }}$ Symposium on Turbulence, Rolla, Missouri, 1992. 
Public reporting burden for this collection of information is estimated to average 1 hour per response, including the time for reviewing instructions, searching existing data sources, gathering and maintaining the data needed, and completing and reviewing the collection of information. Send comments regarding this burden estimate or any other aspect of this collection of information, including suggestions for reducing this burden, to Washington Headquarters Services, Oirectorate for Project (0704-018B). Washington, DC 20503.

\begin{tabular}{l|l|l|} 
1. AGENCY USE ONLY (Leave blank) & 2. REPOAT DATE & 3. REPORT TYPE AND DATES COVERED
\end{tabular}

\begin{tabular}{|l|l|l} 
1. AGENCY USE ONLY (Leave blank) & $\begin{array}{c}\text { 2. REPOAT DATE } \\
\text { June } 1993\end{array}$ & Technical Memorandum
\end{tabular}

4. TITLE AND SUBTITLE

5. FUNDING NUMBERS

Fuzzy Logic Particle Tracking Velocimetry

WU-505-62-50

6. AUTHOR(S)

Mark P. Wernet

7. PERFORMING ORGANIZATION NAME(S) AND ADDRESS(ES)

8. PERFORMING ORGANIZATION REPORT NUMBER

National Aeronautics and Space Administration

Lewis Research Center

E-7900

Cleveland, Ohio 44135-3191

10. SPONSORING/MONITORING

9. SPONSORING/MONITORING AGENCY NAME(S) AND ADDRESS(ES) AGENCY REPORT NUMBER

National Aeronautics and Space Administration

Washington, D.C. 20546-0001

NASA TM-106194

\section{SUPPLEMENTARY NOTES}

Prepared for the Optical Diagnostics in Fluid and Thermal Flow sponsored by the Society of Photo-Optical Instrumentation Engineers, San Diego, Califomia, July 11-16, 1993. Responsible person, Mark P. Wernet, (216) 433-3752.

12:. DISTRIBUTION/AVALABILITY STATEMENT

12b. DISTRIBUTION CODE

Unclassified - Unlimited

Subject Category 35

\section{ABSTRACT (Maximum 200 words)}

Fuzzy logic has proven to be a simple and robust method for process control. Instead of requiring a complex model of the system, a user defined rule base is used to control the process. In this paper the principles of fuzzy logic control are applied to Particle Tracking Velocimetry (PTV). Two frames of digitally recorded, single exposure particle imagery are used as input. The fuzzy processor uses the local particle displacement information to determine the correct particle tracks. Fuzzy PTV is an improvement over traditional PTV techniques which typically require a sequence $(>2)$ of image frames for accurately tracking particles. The fuzzy processor executes in software on a PC without the use of specialized array or fuzzy logic processors. A pair of sample input images with roughly 300 particle images each results in more than 200 velocity vectors in under 8 seconds of processing time.

14. SUBJECT TERMS

Velocity measurement; Particle trajectory; Laser velocimetry

17. SECURTY CLASSIFICATION OF REPORT Unclassified

18. SECURITY CLASSIFICATION
OF THIS PAGE
Unclassified

Unclassified
19. SECURITY CLASSIFICATION OF ABSTRACT Unclassified 